\title{
Outbreak of Zika virus disease
} in the Americas and the association with microcephaly, congenital malformations and Guillain-Barré syndrome

\author{
Shamez N Ladhani, ${ }^{1,2}$ Catherine O'Connor, ${ }^{1}$ Hilary Kirkbride, ${ }^{1}$ \\ Tim Brooks, ${ }^{3}$ Dilys Morgan ${ }^{1}$
}

\section{INTRODUCTION}

Prior to 2007, Zika virus (ZIKV) was generally considered an arbovirus of limited importance, causing a mild self-limiting febrile illness in tropical Africa and Southeast Asia. Now, a large, ongoing outbreak of ZIKV that started in Brazil in early 2015 is spreading rapidly across the Americas and has been potentially linked to congenital malformations (including microcephaly) and Guillain-Barré syndrome (GBS). In England, as of 4 February 2016, five adults have been diagnosed with ZIKV infection following travel to countries currently experiencing a ZIKV outbreak.

\section{ZIKA VIRUS}

ZIKV was first isolated from a monkey employed as a sentinel animal in a yellow fever study in the Zika forest, near Entebbe, Uganda, in $1947 .{ }^{1} \mathrm{ZIKV}$ is an RNA arbovirus belonging to the Flaviviridae family, which also includes dengue, Japanese encephalitis and West Nile viruses. The virus is transmitted by female Aedes mosquitoes, especially, Aedes aegypti, which is also an effective vector of dengue and chikungunya virus. Unlike many other mosquito vectors (eg, Anopheles spp. that transmit malaria), Aedes are predominantly day-biting mosquitoes. While the majority of human infections with ZIKV are likely to be acquired via mosquitoes, the virus has been detected in semen ${ }^{2}$ and blood donors who were asymptomatic at the time of donation, ${ }^{3}$ raising the possibility of sexual transmission and transmission through blood transfusion, respectively.

\footnotetext{
${ }^{1}$ National Infection Service, Public Health England, London, UK; ${ }^{2}$ Paediatric Infectious Diseases Research Group, St. George's University of London, London, UK; ${ }^{3}$ Rare and Imported Pathogens Laboratory, Public Health England, Salisbury, UK

Correspondence to Dr Shamez N Ladhani, Immunisation Department, Public Health England, 61 Colindale Avenue, London NW9 5EQ, UK; shamez.ladhani@phe.gov.uk
}

\section{CLINICAL DISEASE}

Up to $80 \%$ of individuals infected with ZIKV remain asymptomatic and the remainder usually develop a mild selflimiting febrile illness lasting 4-7 days associated with maculopapular rash, arthralgia, conjunctivitis, itching, myalgia and headache. The infection is seldom severe enough to warrant hospitalisation and ZIKV-related deaths are very rarely reported and are mostly associated with underlying comorbidities. Most recently, a teenager with sickle cell disease in Colombia died after developing acute respiratory distress syndrome and hepatic necrosis. ${ }^{4}$ There is no vaccine to prevent ZIKV infection and there are no specific antiviral treatments available.

\section{EPIDEMIOLOGY}

As ZIKV generally causes such a mild selflimiting illness, knowledge of its geographical distribution is dependent mainly on individual case reports, outbreaks and seroprevalence studies. Prior to 2007, reports of ZIKV infection were restricted to African and Asian countries. The first major outbreak outside the ZIKV natural territory was reported in 2007 on Yap Island in Micronesia. In 2013, the virus spread further east to cause large outbreaks in French Polynesia with subsequent spread to New Caledonia, the Cook Islands and Easter Island.

In 2015, ZIKV underwent its largest and fastest geographical expansion. The first autochthonous cases of ZIKV on continental America were confirmed in northeastern Brazil in May 2015 and, since then, more than 20 countries and territories in the Americas have reported local transmission, with the number of affected countries increasing every week (see regular Pan American Health Organization (PAHO) updates for latest affected countries). A concurrent outbreak is also currently being reported from Cape Verde and, occasionally, cases are diagnosed in travellers returning from African and Asian countries.

\section{ZIKV AND MICROCEPHALY WITH CONGENITAL MALFORMATIONS}

In October 2015, an unprecedented increase in cases of infants with microcephaly (box 1), temporally associated with the emergence of ZIKV, was reported in the northern Brazilian state of Pernambuco. Retrospective analysis of the Brazilian live birth information system identified several Brazilian states with significant increases in microcephaly compared with previous years. This increase has continued and, as of 3 February 2016, 4783 cases of microcephaly across Brazil have been notified, compared with the expected 150-200 annual cases (see Brazilian Ministry of Health for more information). A large proportion (3670/ $4783,77 \%)$ is still under investigation and awaiting confirmation. So far, 709 $(15 \%)$ cases have been discarded and, of the $404(8 \%)$ confirmed with microcephaly, 17 (4\%) have tested positive for ZIKV.

In a cohort of 35 Brazilian infants with microcephaly born during AugustOctober 2015 and reported to the registry, all the mothers lived in or had visited

Box 1 Surveillance case definition used by the Brazilian authorities for congenital microcephaly

- Pregnant women with acute exanthema, and excluded other known infectious and non-infectious possible causes

- Fetuses with ultrasonographic head circumference of $>2$ SDs below the mean for gestational age, with or without associated neurological malformations

- Fetuses with ultrasonographic neurological findings suggestive of intrauterine infection

- Spontaneous abortions in women with an exanthem illness without any identified cause during pregnancy

- Stillbirths at any gestational age in women with an exanthem illness without any identified cause during pregnancy

- Live newborns born prematurely (<37 weeks gestation) with a head circumference below the third percentile

- Live term newborns with a head circumference $\leq 32 \mathrm{~cm}$ 
ZIKV-affected areas during pregnancy, 25 (71\%) infants had severe microcephaly (head circumference $>3$ SD below the mean for sex and gestational age), 17 (49\%) had at least one neurological abnormality and all 27 infants who underwent neuroimaging had abnormalities. $^{5}$ While the clinical characteristics and outcomes in these children are only beginning to be characterised, the current evidence strongly indicates a causal link (see European Centre for Disease Control and Prevention (ECDC) website for summary). ZIKV appears to share many similarities to rubella (asymptomatic infection or mild illness with fever and rash, but potential for serious birth defects if infected during pregnancy) but not with Ebola virus (a severe, haemorrhagic infection with a high case fatality rate, that is transmitted from wild animals and spread through human-to-human transmission).

An increase in cases of microcephaly has yet to be reported from other countries affected by ZIKV. However, in November 2015, the French Polynesian health authorities retrospectively reported an increase in congenital neurological malformations in fetuses and newborn infants from an average of one annual case to 17 cases during 2014-2015. These malformations consisted of 12 foetal cerebral malformations or multiple congenital malformations, including brain lesions, and five infants with brainstem dysfunction and absence of swallowing. Pregnancy was terminated in nine cases. The remaining infants were born at term with normal body measurements, but were found to have multiple malformations and brain lesions. Common congenital infections were excluded and further investigations are ongoing. None of the mothers reported symptoms of ZIKV infection during pregnancy. Based on the temporal correlation of these cases with the ZIKV outbreaks in French Polynesia, the local health authorities hypothesise that ZIKV infection may be associated with neurological abnormalities if mothers are infected during the first or second trimester of pregnancy (see ECDC website for summary).

\section{ZIKV AND GBS}

In addition to microcephaly and congenital malformations, a temporal association between ZIKV infection and an increase in cases of GBS is currently being observed in Brazil, El Salvador and Venezuela. GBS was also reported following the ZIKV outbreak in French Polynesia.

\section{ZIKV RISK IN THE UK AND EUROPE}

ZIKV is likely to continue to spread across the Americas in the coming months, with the exception of Canada and continental Chile, the only countries in the Americas where Aedes mosquitoes are not present. A number of European and other countries have already reported imported cases of ZIKV. With the continuing spread of the infection in the Americas, along with increasing awareness among travellers and clinicians, it is likely that more cases will be diagnosed and reported in the coming months in returning travellers. As of 4 February 2016, five cases have been diagnosed in the UK in travellers who have recently returned from the Americas (see Public Health England (PHE) website for further updates). The risk of ZIKV becoming established in the UK is currently negligible due to a lack of a competent vector. However, the risk of potential onward transmission of ZIKV in some southern European countries where Aedes are present is a real concern as we move into the spring and summer months when mosquito vectors become more active.

\section{IMPLICATIONS FOR TRAVELLERS}

As neither treatment nor vaccines are available, prevention of ZIKV infection relies on personal protection measures against mosquito bites, similar to dengue, chikungunya and other mosquitoborne infections. Pregnant women and those trying to become pregnant are advised to consider avoiding travel to affected areas. If travel is unavoidable, then strict preventive measures must be taken to avoid mosquito bites, both during daytime and night-time hours (but especially during mid-morning and late afternoon to dusk, when the mosquito is most active). Travellers showing symptoms compatible with dengue, chikungunya or ZIKV infection during travel or within 3 weeks of returning from an affected area should seek medical advice. Pregnant women who have travelled to areas with active ZIKV transmission should mention their travel history during antenatal visits in order to be assessed and monitored appropriately.

\section{IMPLICATION FOR PAEDIATRICIANS AND NEONATOLOGISTS}

1. Paediatricians should be aware of the mosquito-borne ZIKV outbreak in the Americas.

2. The vast majority of ZIKV infections are asymptomatic (80\%) or selflimiting, with most individuals developing a mild self-limiting febrile illness lasting 4-7 days associated with maculopapular rash, arthralgia, conjunctivitis, myalgia and headache. However, ZIKV infection can potentially be severe in those with underlying medical conditions.

3. Infection with ZIKV in pregnancy is potentially associated with microcephaly and congenital malformations, particularly those of the central nervous system.

4. ZIKV has also been associated with GBS

5. ZIKV should be considered in the differential diagnosis of:

A. any patient with fever returning from a country where ZIKV outbreaks are ongoing (see National Travel Health Network and Centre ( $\mathrm{NaTHNaC}$ ) website for further updates);

\section{Useful websites for further information}

- WHO: http://www.who.int/mediacentre/factsheets/zika/en/

- PAHO: http://www.paho.org/hq/index.php?option=com_topics\&view=article\&id= 427\&Itemid $=41484$

- Brazilian Ministry of Health: http://combateaedes.saude.gov.br

- ECDC: http://ecdc.europa.eu/en/healthtopics/zika_virus_infection/Pages/index.aspx

- US Center for Disease Control and Prevention (CDC): http://www.cdc.gov/zika/

- US CDC interim guidance for the evaluation and testing of infants with possible congenital ZIKV infection: http://www.cdc.gov/mmwr/volumes/65/wr/mm6503e3. htm\#B1_down

- Public Health England: https://www.gov.uk/guidance/zika-virus\#zika-in-the-uk

- NaTHNaC: http://travelhealthpro.org.uk/

- Rare and Important Pathogens Laboratory, Public Health England: https://www. gov.uk/government/collections/rare-and-imported-pathogens-laboratory-ripl 
B. any neurological and autoimmune syndromes (including GBS) in a patient who has recently travelled to a country where ZIKV outbreaks are ongoing;

C. miscarriage, stillbirth, in-utero death due to infection, congenital infection, microcephaly or any other congenital (especially neurological) abnormality in any infant born to a mother who travelled to a country where ZIKV outbreaks are ongoing at any time during pregnancy, even if the mother was asymptomatic throughout her pregnancy.

\section{INVESTIGATION AND MANAGEMENT OF SUSPECTED ZIKV}

- ZIKV infection can be confirmed by direct detection of ZIKV RNA or specific viral antigens in clinical specimens.

- If a case of ZIKV is suspected, clinical advice and information about diagnostic testing should be sought in the first instance by contacting the local virologist, microbiologist or infectious disease consultant. The Rare and Imported Pathogens Laboratory can provide further specialist advice as required (https://www. gov.uk/government/collections/rare-andimported-pathogens-laboratory-ripl).

- Public Health England advice on travel, assessment of pregnant women following travel, advice for those working in primary care, deferral of blood donation, information for the public about ZIKV and media enquiries are available online (see PHE website for further updates).

- Guidance on the investigation and management of infants born to mothers who traveled to a ZIKV endemic country during pregnancy will shortly be available on the PHE website.

- A British Paediatric Surveillance Unit is planned to monitor adverse outcomes in infants born to mothers who traveled to a ZIKV endemic country.

Contributors SNL and CO wrote the first draft of the paper. All authors contributed to the final, submitted manuscript.

Competing interests None declared.

Provenance and peer review Commissioned; internally peer reviewed.
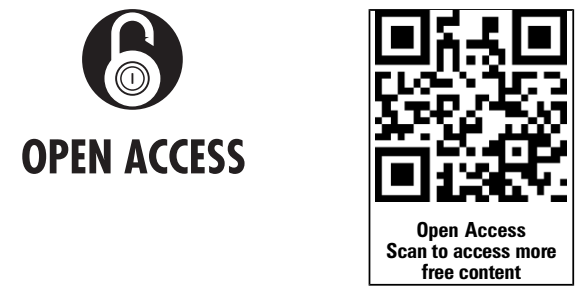

Open Access This is an Open Access article distributed in accordance with the Creative Commons Attribution Non Commercial (CC BY-NC 4.0) license, which permits others to distribute, remix, adapt, build upon this work non-commercially, and license their derivative works on different terms, provided the original work is properly cited and the use is noncommercial. See: http://creativecommons.org/licenses/ by-nc/4.0/

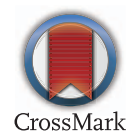

To cite Ladhani SN, O'Connor C, Kirkbride $\mathrm{H}$, et al. Arch Dis Child 2016;101:600-602.

Received 1 February 2016

Revised 8 February 2016

Accepted 14 February 2016

Published Online First 14 March 2016

Arch Dis Child 2016;101:600-602.

doi:10.1136/archdischild-2016-310590

\section{REFERENCES}

1 Dick GW, Kitchen SF, Haddow AJ. Zika virus. I. Isolations and serological specificity. Trans R Soc Trop Med Hyg 1952;46:509-20.

2 Musso D, Roche C, Robin E, et al. Potential sexual transmission of Zika virus. Emerg Infect Dis 2015:21:359-61.

3 Musso D, Nhan T, Robin E, et al. Potential for Zika virus transmission through blood transfusion demonstrated during an outbreak in French Polynesia, November 2013 to February 2014. Euro Surveill 2014;19:pii: 20761.

4 Arzusa-Ortega L, Polo A, Pérez-Tatis G, et al. Fatal Zika virus infection in girl with sickle cell disease, Colombia. Emerging Infectious Diseases 2016. http:// wwwnc.cdc.gov/eid/article/22/5/15-1934_article

5 Schuler-Faccini L, Ribeiro EM, Feitosa IM, et al, Brazilian Medical Genetics Society-Zika Embryopathy Task Force. Possible association between Zika virus infection and microcephaly — Brazil, 2015. MMWR Morb Mortal Wkly Rep 2016;65:59-62. 